\title{
Intensive insulin therapy in the intensive care unit
}

\author{
Greet Van den Berghe MD PhD, Dieter Mesotten MD PhD, Ilse Vanhorebeek PhD
}

Published at www.cmaj.ca on Mar. 24, 2008.

$\infty \quad$ See related research paper by Griesdale and colleagues, page 821

$\mathrm{H}$ yperglycemia in response to critical illness has long been associated with adverse outcomes. Large observational studies have recently confirmed and fine-tuned this association, reporting the lowest risk of death with blood glucose levels roughly between 5 and $7 \mathrm{mmol} / \mathrm{L}$ or between 6 and $8 \mathrm{mmol} / \mathrm{L}$ for patients with diabetes mellitus. ${ }^{1,2}$ This association is thought to be causal based on potential glucose toxicity in the context of ischemia-reperfusion. Studies in patients and animals support the hypothesis of toxicity induced by excess glucose, specifically toxicity to the mitochondria of cells that take up glucose independent of insulin and in proportion to the circulating levels of glucose..$^{3-5}$ However, the level at which blood glucose becomes toxic in the clinical context of critical illness is unclear.

Several studies with heterogeneous designs and outcome measures have tested the hypothesis that tight control of blood glucose levels improves outcomes in critically ill patients. The first such randomized trial targeted a "strictly normal" level for fasting glucose (i.e., 4.4-6.1 mmol/L), compared with usual care, in adult patients in surgical intensive care units (ICUs). ${ }^{6}$ Usual care, at that time, was to initiate insulin only when a patient's blood glucose level exceeded the renal threshold $(12 \mathrm{mmol} / \mathrm{L})$ and to discontinue insulin when the level decreased below $10 \mathrm{mmol} / \mathrm{L}$. The intervention comprised insulin infused continuously via an accurate syringe-driven infusion pump to patients in a nonfasting state. Dose modifications were performed frequently by well-trained bedside nurses who used accurate blood gas analyzers to measure glucose and potassium levels. The intervention was labelled "intensive insulin therapy" and was found to reduce morbidity and mortality. A subsequent study in a medical ICU setting ${ }^{7}$ documented similar organ protective effects, although not as strikingly as in the surgical ICU study. Mortality was not different in an intention-to-treat analysis in the second study.

In this issue of $C M A J$, Griesdale and colleagues ${ }^{8}$ report a meta-analysis of 26 studies involving over 13500 patients. The authors concluded that intensive insulin therapy does not provide an overall mortality benefit, but that it may decrease mortality among surgical ICU patients. The differences among the individual studies are likely important. First, the type of patients and type and logistics of the intensive care 8 units differed substantially. Second, the accuracy of insulin infusion and glucose monitoring differed among studies. In the initial randomized trial, ${ }^{6}$ all of the patients had central venous lines for reliable adjustment of continuous insulin infusion, and arterial lines for monitoring glucose and potassium, thus avoiding hypokalemia-induced arrhythmia. In several subsequent studies, insulin was administered as peripheral ve-

\section{Key points}

- Hyperglycemia and hypoglycemia are linked to adverse outcomes in different types of patients with critical illness.

- Lowering blood glucose with insulin does not affect mortality in all critically ill patients.

- Intensive insulin therapy may be effective in lowering the risk of death among surgical patients in intensive care units.

- Differences in study outcomes may be because of the duration of hyperglycemia before the start of the intervention, the target blood glucose ranges in the 2 groups, the accuracy of blood glucose measurement and the degree of success in reaching the target level while avoiding hypoglycemia.

- Further research is needed to identify the optimal target level for blood glucose in different patient populations and to improve the tools for implementation in clinical practice.

nous or subcutaneous infusions (including bolus injections), measurement of blood glucose was often infrequent, and sampling was performed via finger pricks with glucometers that were often insufficiently accurate for the intensive care setting. ${ }^{9}$ Furthermore, the blood glucose targets for the intervention and comparator groups differed among studies. Most importantly, success in achieving these targets varied greatly among the included studies; the experience of the nursing team may have played a role in these differences.

When used in such different ways, the term "intensive insulin therapy" may not always describe the same intervention. In some settings, such therapy may have induced large fluctuations in blood glucose, possibly with undetected hypokalemia and hypoglycemia alternating with hyperglycemia. This might have resulted in a reduced average blood glucose level; however, the fluctuation in blood glucose may be worse than constant moderate hyperglycemia.

Such differences may explain the finding that patients in surgical ICUs may benefit from intensive insulin therapy more than patients in medical ICUs. First, surgical ICUs routinely use central venous and arterial lines and are able to monitor and correct glucose and potassium more accurately. This may not be the case in medical ICUs, in which patients often do not have arterial and central lines and glucometers may be used for measurement of glucose via finger pricks, which have been shown to be inaccurate ${ }^{9}$ and may lead to dosing errors.

Greet Van den Berghe, Dieter Mesotten and Ilse Vanhorebeek are with the Department and Laboratory of Intensive Care Medicine, Catholic University of Leuven, Leuven, Belgium. 
Second, hyperglycemia in surgical ICU patients is usually triggered by surgery or trauma, which is the equivalent of acute ischemia-reperfusion, and, thus, the delay between onset of hyperglycemia and the start of glycemic control is usually short. In contrast, patients in medical ICUs often have suffered from chronic illnesses before admission and hyperglycemia was more likely to have been present for a longer time in these patients. This may have induced adaptive changes to protect the cells against elevated extracellular glucose such that acute lowering of blood glucose may not be beneficial. Alternatively, the time window for the prevention of glucose toxicity may have passed. Evidence supporting this hypothesis comes from randomized trials of tight glucose control in critical illness in adults, ${ }^{10}$ and recently in children ${ }^{11}$ and patients with type 2 diabetes. ${ }^{12,13}$

Third, the meta-analysis suggests that most studies in surgical ICUs managed to achieve the target blood glucose level, whereas studies in medical or mixed ICUs often did not. It appears that the studies that did not succeed in reaching the target reported no benefit or increased mortality, whereas the studies that succeeded in reaching the strict normoglycemia target in most patients showed reduced mortality.

The term intensive insulin therapy is used to refer to different practices involving lowering glucose in patients in ICUs. Whether insulin therapy should be given to all patients in ICUs is not the question to ask. Instead, we need to ask how cellular toxicity caused by glucose levels that are higher than the patient's premorbid levels can be avoided and what the window of opportunity is for doing so. Further exploratory meta-analysis and better focused randomized intervention studies are needed to answer this question.

What should clinicians do today, given the results of the study by Griesdale and colleagues? Unfortunately, the evidence does not allow confident recommendations for an overall guideline, because the optimal target for glucose in different groups of critically ill patients is unknown. Hence, clinicians should consider the following criteria: the level of evidence of the individual studies, particularly whether the hypothesized benefit was realistic, whether power was sufficient to detect this effect, whether the tools to measure and control blood glucose were adequate, whether the targets were reached, and whether the comparator level of glycemic control was relevant. If the above criteria are satisfied, clini- cians should then assess how comparable the patients in these studies are to their own patients. Currently, the evidence is strongest for adults and children in surgical ICUs. For patients considered eligible for tight glucose control, the clinician should assess whether the intervention can be applied safely in the specific clinical setting.

This article has been peer reviewed.

Competing interests: None declared.

Contributors: Greet Van den Berghe wrote the first draft of the manuscript. All of the authors revised the manuscript for intellectual content and approved the version submitted for publication.

\section{REFERENCES}

1. Kosiborod M, Inzucchi SE, Krumholz HM, et al. Glucometrics in patients hospitalized with acute myocardial infarction: defining the optimal outcomes-based measure of risk. Circulation 2008;117:1018-27.

2. Bagshaw SM, Egi M, George C, et al.; for the ANZICS Database Management Committee. Early blood glucose control and mortality in critically ill patients in Australia. Crit Care Med 2009 Dec 26. [Epub ahead of print]

3. Van den Berghe G. How does blood glucose control with insulin save lives in intensive care? J Clin Invest 2004;114:1187-95.

4. Langouche L, Vanhorebeek I, Vlasselaers D, et al. Intensive insulin therapy protects the endothelium of critically ill patients. J Clin Invest 2005;115:2277-86.

5. Vanhorebeek I, De Vos R, Mesotten M, et al. Protection of hepatocyte mitochondrial ultrastructure and function by strict blood glucose control with insulin in critically ill patients. Lancet 2005;365:53-9.

6. Van den Berghe G, Wouters P, Weekers F, et al. Intensive insulin therapy in critically ill patients. N Engl J Med 2001;345:1359-67.

7. Van den Berghe G, Wilmer A, Hermans G, et al. Intensive insulin therapy of medical intensive care patients. N Engl J Med 2006;354:449-61.

8. Griesdale DE, de Souza RJ, van Dam RM, et al. Intensive insulin therapy and mortality among critically ill patients: a meta-analysis including NICE-SUGAR study data. CMAJ 2009;180:821-7.

9. Scott MG, Bruns DE, Boyd JC, et al. Tight glucose control in the intensive care unit: Are glucose meters up to the task? Clin Chem 2009;55:18-20.

10. Van den Berghe G, Wilmer A, Milants I, et al. Intensive insulin therapy in mixed medical/surgical ICU: benefit versus harm. Diabetes 2006;55:3151-9.

11. Vlasselaers D, Milants I, Desmet L, et al. Intensive insulin therapy in paediatric intensive care unit patients: a prospective randomized controlled study. Lancet 2009:373:547-56.

12. The Action to Control Cardiovascular Risk in Diabetes Study Group (ACCORD) Effects of intensive glucose lowering in type 2 diabetes. $N$ Engl J Med 2008;358:2545-59.

13. The ADVANCE Collaborative Group. Intensive blood glucose control and vascular outcomes in patients with type 2 diabetes. N Engl J Med 2008;358:2560-72.

Correspondence to: Dr. Greet Van den Berghe, Intensive Care Medicine, Division of Acute Medical Sciences, Herestraat 49, Leuven B-3000, Belgium; fax 32-16-34-40-15;

greet.vandenberghe@med.kuleuven.be 